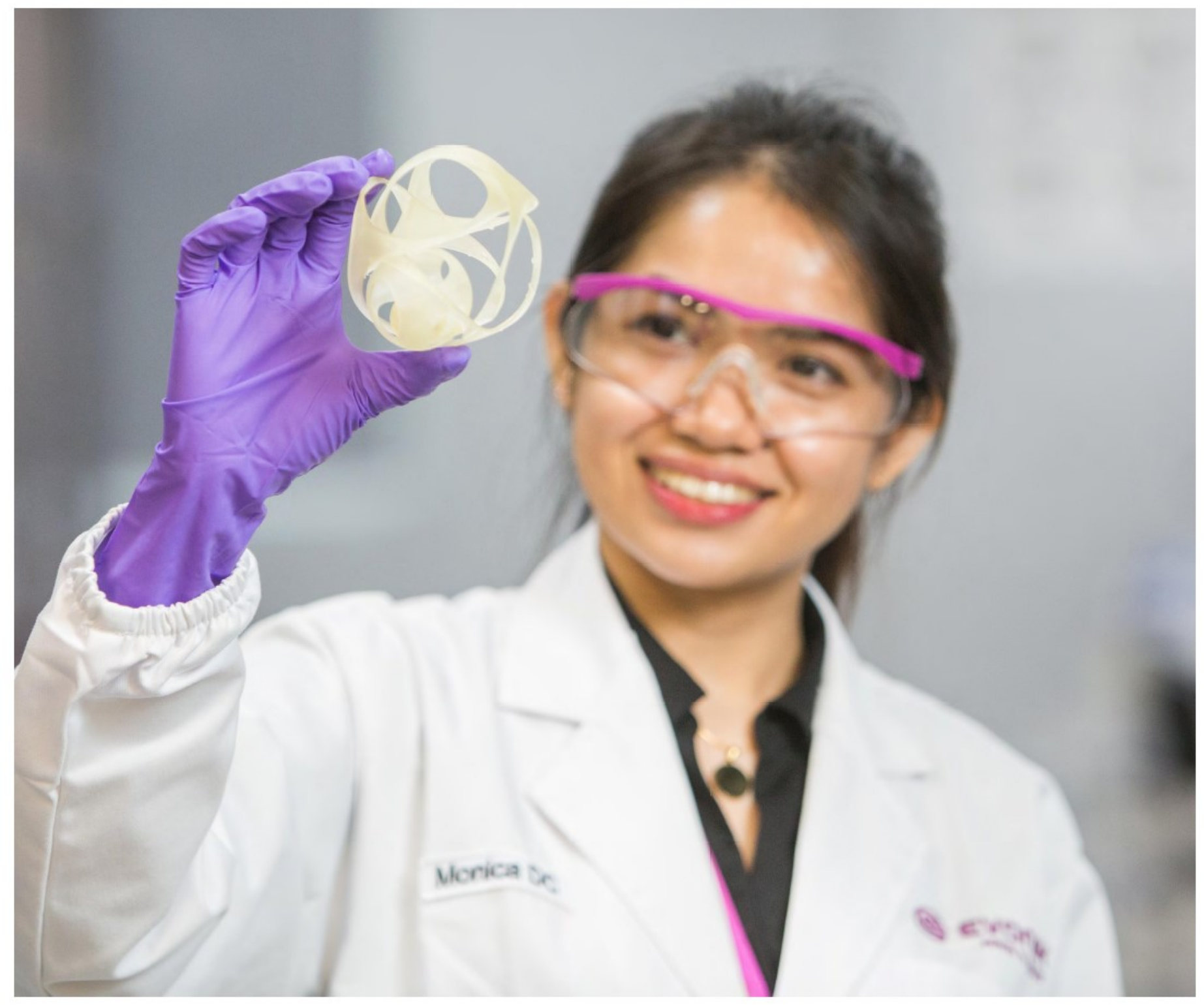

\section{Die Grenzen der} Chemie neu ausloten? It takes \#HumanChemistry
Wir suchen kreative Chemikerinnen und Chemiker, die mit uns gemeinsam neue Wege gehen wollen mit Fachwissen, Unternehmertum und Kreativität für innovative Lösungen. Informieren Sie sich unter:

evonik.de/karriere 


\title{
Gaining Insight into the Deformation of Achilles Tendon Entheses in Mice
}

\author{
Julian Sartori,* Sebastian Köhring, Stefan Bruns, Julian Moosmann, \\ and Jörg U. Hammel**
}

Understanding the biomechanics of tendon entheses is fundamental for surgical repair and tissue engineering but also relevant in biomimetics and paleontology. Examinations into the 3D tissue deformation under load are an important element in this process. However, entheses are difficult objects for microcomputed tomography due to extreme differences in X-ray attenuation. Herein, the ex vivo examination of Achilles tendon entheses from mice using a combination of tensile tests and synchrotron radiation-based microcomputed tomography is reported. Strains and volume changes are compared between the more proximal free tendon and the distal tendon that wraps around the Tuber calcanei.

Tomographic datasets of relaxed and deformed entheses are recorded with propagation-based phase contrast. The tissue structure is rendered in sufficient detail to enable manual tracking of patterns along the tendon, as well as digital volume correlation in a suitable pair of tomographic datasets. The strains are higher in the distal than in the proximal tendon. These results support the existence of a compliant zone near the insertion. Necessary steps to extend the automatic tracking of tissue displacements to all stages of the deformation experiment are discussed.

\section{Introduction}

In the locomotor system, bones mainly provide bending and compression stiffness, ${ }^{[1]}$ whereas tendons and ligaments transfer tensile loads. ${ }^{[2]}$ These load cases merge at insertions of tendons and ligaments into bone, called entheses. Insertions to the ends of long bones usually share a characteristic transition in tissue composition, structure and properties, termed the fibrocartilaginous enthesis. ${ }^{[3-8]}$ The collagen fibers pass through unmineralized and mineralized fibrocartilage before they separate into fibrils that seem to be continuous with the collagen fibrils of bone. ${ }^{[9]}$

There is a long record of examinations into the tensile behavior of tendons and ligaments. The diversity of methods used and structures examined is vast. We give a gross summary of studies that include a comparison of the midsubstance and the insertion. Commonly, higher strains and lower moduli are found near the insertion than in the midsubstance. Only a few studies in intact tendons find the highest strains in or towards the tendon midsubstance. ${ }^{[10,11]}$ Several studies in intact tendons ${ }^{[12-14]}$ find strains at the insertion to be about twice as high as in the midsubstance - one found a higher difference. ${ }^{[15]}$ Examinations in which the specimens were cut generally tend to find more pronounced differences between a stiff tendon or ligament and a compliant insertion. ${ }^{[5,8,16-18]}$ Only some studies of the biomechanics of tendon-bone insertions are based on high resolution imaging: By a combination of X-ray dispersive spectroscopy and tensile tests in microscopic beams, Deymier et al. ${ }^{[7]}$ were able to identify a region within the mineralization gradient that is more compliant than the neighboring unmineralized fibrocartilage. Four other studies also confirmed the existence of a very localized compliant zone. ${ }^{[8,17,19,20]}$ Rossetti et al. ${ }^{[8]}$ also found that the behavior of the unmineralized fibrocartilage is very heterogeneous within a narrow band neighboring the mineralization front, a region in which the fibers at the same time contain more collagen type II.

The reviewed publications suggest that a zone with a higher compliance than either bone or tendon is present at many insertions. Recent findings and models support the notion that tendons and fibrocartilages are poroelastic. ${ }^{[21-23]} \mathrm{A}$ mechanical model of wrap-around tendons ${ }^{[24]}$ suggests that a lower permeability in compressed regions and pressurization of the liquid phase could reduce stress in the solid phase. Slicing significantly 
alters tissue biomechanics by generating an open surface where pressurized liquid can evade. ${ }^{[25]}$ Therefore, it is not yet clear, whether the findings of high strains at the insertion have to be partly attributed to the destruction of tissue structure by slicing.

A further finding that is in contrast with an adaptionist expectation of enthesis mechanics was recently added by Sevick et al. ${ }^{[25]}$ who reported that fibers change their angle of insertion under load. Accordingly, the fibers are bent within a narrow band near the mineralization front. Schneider ${ }^{[26]}$ delineated how such localized bending could lead to increased stress. Sevick et al. reported their findings on the basis of observations in sliced specimens. However, a pressurization of incompressible liquid in the matrix between the fibers could protect the fibers from high curvatures near the mineralization front. ${ }^{[27]}$ Therefore, a re-examination of the deformation of entheses in intact specimens is worthwhile.

A re-examination of enthesis deformation should be based on 3D imaging to account for the "subsurface nature of interfacial tissue.". ${ }^{28]}$ The tensile response of tendon is determined by microscopic deformation mechanisms such as fiber realignment and sliding. ${ }^{[12,29]}$ Therefore, a resolution of few micrometers is required over a field of view spanning the tendon enthesis to relate the macroscopic biomechanics to the deformation of microscopic structure.

Magnetic resonance imaging (MRI) is the most common method to examine in vivo tissue behavior, but it does not reach the required resolution. ${ }^{[8,30]}$ Microscopy is limited with regard to penetration depth even if multiphoton techniques are used. ${ }^{[31]} \mathrm{In}$ microcomputed tomography, the low X-ray attenuation of soft tissues is challenging. This is complicated by the immediate neighborhood of soft and hard tissues within entheses. The most common means for visualizing soft tissue structure are disadvantageous in deformation studies. Contrast agents alter the mechanical properties of the treated tissues. ${ }^{[32,33]}$
Propagation-based phase-contrast is achieved at the cost of long acquisition times in bench-top and laboratory devices, ${ }^{[34,35]}$ whereas deformation studies necessitate rapid imaging to minimize motion blur caused by the relaxation of soft tissue under load.

Synchrotron radiation-based micro computed tomography (SR $\mu \mathrm{CT}$ ) exhibits suitable properties for 3D examinations of soft tissue deformation. It provides the required spatial resolution and field of view. Untreated soft tissue samples can be imaged using grating- or propagation-based phase contrast because of the coherence of synchrotron radiation as exemplified by recent studies. ${ }^{[36,37]}$ Short tomography times are reached because of its brilliance. ${ }^{[13,29]} 3 \mathrm{D}$ strains at high resolutions for the full volumes can be derived from the resulting data by digital volume correlation (DVC). ${ }^{[37-39]}$

This study provides the first images at a microscopic resolution of the tissue deformation inside an intact tendon enthesis under load. The parameter search for the specimen environment, the mechanical tests and the imaging was carried out together with the acquisition of the reported image data.

Mouse hind limbs (Figure 1A) were dissected to expose the calf muscle (M. triceps surae) and the Achilles tendon. Each specimen was mounted in a custom tensile testing device (Figure 1B,C). One group of specimens $(n=3)$ was rinsed with deionized water after mounting, one group did not receive further treatment. The initial tomographic dataset was recorded in a relaxed state in which a small preload was applied to the Achilles tendon. The second dataset was recorded in a quasi-static state after application of a physiological load and a relaxation time. If the specimen did not fail in the next loading step, a third dataset was recorded. Various measures, described below, are used to quantify tissue response to load. Critical parameters for achieving a more realistic mechanical testing, higher temporal resolution and enhanced image quality are reported to facilitate future investigations into enthesis deformation.
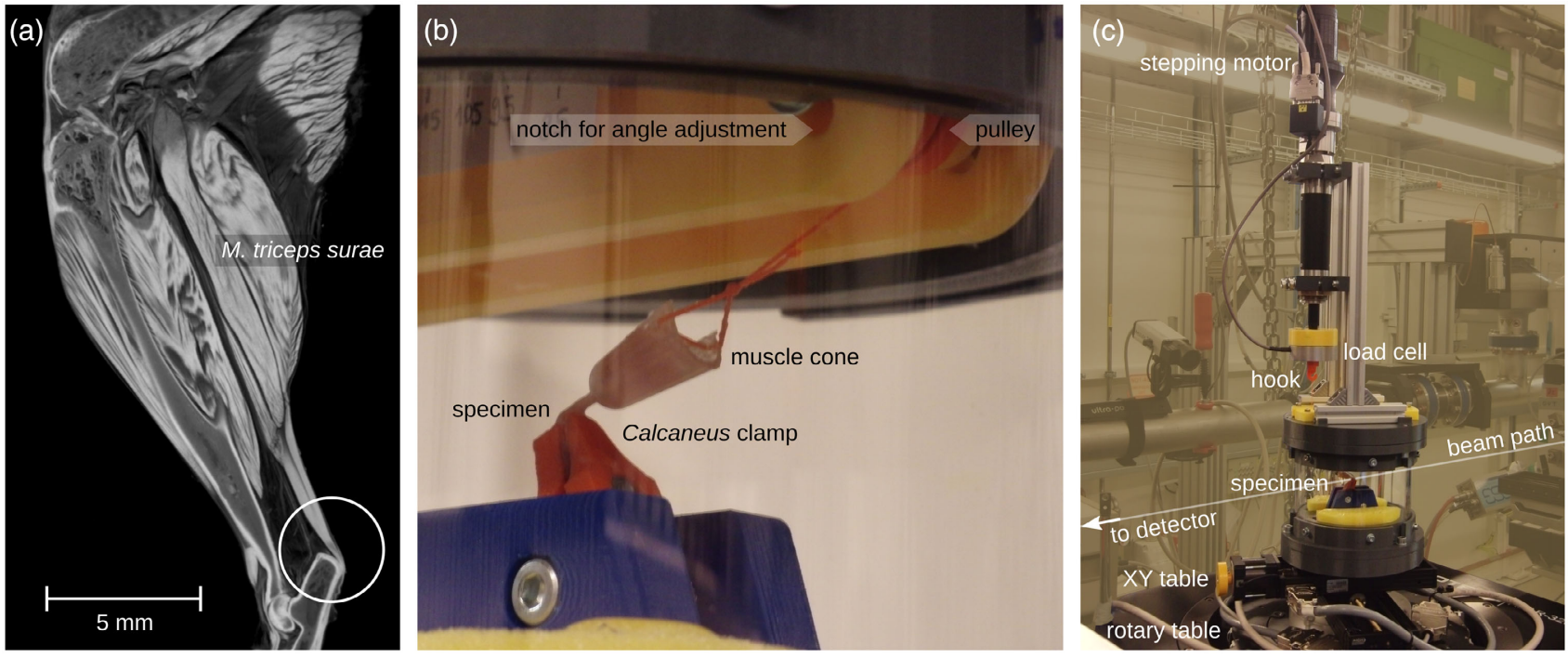

Figure 1. Specimen anatomy and experimental setup. A) Volume rendering of the lower left limb of a mouse virtually cut by a sagittal section plane. The circle marks the Achilles tendon and its insertion at the Calcaneus. B) A specimen is mounted in the tensile testing chamber, the M. triceps surae is trapped in a cone and the Calcaneus is clamped. C) The tensile testing device allows remote control of displacement and logging of forces throughout several synchrotron radiation-based microcomputed tomographies. The region of interest is positioned over the rotation axis by an XY table. 


\section{Results}

\subsection{Forces, Relaxation, and Rupture}

Specimens withstood a force program of one to four force steps. Most often they failed when the force was increased to force level three after the third tomography. In tests with a higher target force in the first force step, failure often occurred during the second force step. Rupture most often occurred at the insertion leaving a smooth surface behind that presumably corresponds to the mineralization front. A control specimen that was not exposed to radiation during the tomography phase did not fail within six force steps (Figure S1, Supporting Information). At the last force level, a target force of $1.65 \mathrm{~N}$ was reached in the control.

The relaxation behavior of the specimens (Figure 2A) can be approximated with logarithmic curves. In unwatered specimens, the mean measured force with standard deviation decreased to $60 \pm 3 \%$ of the target force within the relaxation time at force level one and further to $55 \pm 4 \%$ of the target force within the following tomography phase. At corresponding times in the force program, the mean forces with standard deviation were $62 \pm 4 \%$ to $55 \pm 7 \%$ in the watered specimens. In the control specimen, $66 \%$ and $58 \%$ of the target force were measured.

\subsection{Imaging and Deformation of the Soft Tissues}

Propagation-based phase-contrast imaging renders the hard tissues with surrounding phase-contrast fringes (Figure 2B-E). The Calcaneus is the largest object with a strong attenuation in each specimen. Furthermore, in many specimens mineralizations are found in the free tendon at the proximal end of the dataset. In regions corresponding to beam paths that also passed through hard tissues, the $3 \mathrm{D}$ reconstructed image is noisy. Especially adjacent to the Calcaneus, near the insertion, artifacts are superimposed on the tendon structure. In spite of the artifacts, fiber orientations can be identified. The gray values in tendon tissue are higher than in the surrounding soft tissues. The bursa between the Calcaneus and the tendon is identified by the homogeneous gray value of the contained liquid. The fat pad situated anterior of the tendon and proximal of the bursa exhibits a characteristic cellular structure.

The patterns that were tracked by human subjects correspond to inhomogeneities along the tendon fibers such as cellular lacunae, varying densities, branching patterns and characteristic arrangements of fibers and non-fibrillar components. The search for patterns is complicated by the superimposition of artifacts in position $\mathrm{P} 0$ near the transition to hard tissue and to a lesser degree at position P1 (Figure 3A,B). At position P2, the search radius for correspondences is large because of the larger displacements. In some specimens, tendon mineralizations are useful as markers or for a validation of nearby patterns.

The deformations during the first force step are larger than during the second one. The orientations of the fibers seem to increase during the first force step.

In the relaxed state, the fibers almost follow straight courses across the mineralization front. The mean angular difference with standard deviation between their orientation in the soft tissue directly above the interface and in the mineralized fibrocartilage directly beneath it amounts to $4 \pm 6^{\circ}$. This angle changes by $15 \pm 8^{\circ}$ during the first force step. Due to this change the fibers experience bending within a narrow soft tissue band comprising a few micrometers above the interface (Figure 2D,E).

The tendon cross-sectional area (CSA) at position P1 was $0.218 \pm 0.029 \mathrm{~mm}^{2}$ in watered specimens and $0.177 \pm 0.014 \mathrm{~mm}^{2}$ in unwatered specimens. The difference is not significant. The cross-sectional areas in the unwatered specimens (Figure 2F,G) are higher than in specimens stained with phosphotungstic acid (PTA) from an earlier study. ${ }^{[40]}$ At force level one, the cross-sectional areas are lower than in the relaxed state, but they do not fall below those found in PTA-stained specimens. In all cases the cross-sectional areas increase with distance from the mineralization front.

\subsection{Tensile Data}

The mean forces during the tomography and the resulting stresses at P1 differ widely between the specimens within each of the force levels.

In unwatered specimens, the mean tangent modulus of the first force step is $287 \pm 97 \mathrm{MPa}$ (Figure $2 \mathrm{H}$ ). In watered specimens, the mean tangent modulus of the first force step is significantly lower and amounts to $42 \pm 15 \mathrm{MPa}$. In the single unwatered specimen, that lasted up to the end of the tomography at the second force level, the tangent modulus increases from the first $(356 \mathrm{MPa})$ to the second force step $(722 \mathrm{MPa})$. In watered specimens the tangent modulus as well increases to $151 \pm 55 \mathrm{MPa}$ in the second force step (Figure $2 \mathrm{H}$ and S2, Supporting Information). Accordingly, the first force step still comprises a part of the toe region of the stress-strain curve in all specimens with two force steps. The results from the watered specimens are not discussed in more detail here because the modulus values demonstrate that their treatment led to profound changes in their material properties.

\subsection{Differences Between Distal End and Free Tendon}

Occasionally, the regional strain measurements along the proximal and the distal tendon yielded negative strain values (Figure S3 and S4, Supporting Information). Such a finding occurred at each of the force levels. At force level one, significantly higher strains were measured in the distal region as compared to the proximal region (Figure 3C and S3, S4, Supporting Information). The mean relative volume loss in the distal tendon seems to be higher than in the proximal tendon (Figure 3D,E), but no significant differences are found at the sample size examined.

\subsection{Findings From Digital Volume Correlation}

With only low contrast available in the soft tissue, a global optimum for the displacement field was not found in the larger deformations of the first force step, but a plausible result was provided for the second force step. In addition, Green-Lagrange strain tensors (Figure 4) were calculated from the displacement field.

Results from DVC and visual analysis were compared in one unwatered specimen with regard to the linear strain along the measuring distance used in the visual analysis. Qualitatively, DVC and visual analysis consistently lead to the measurement 

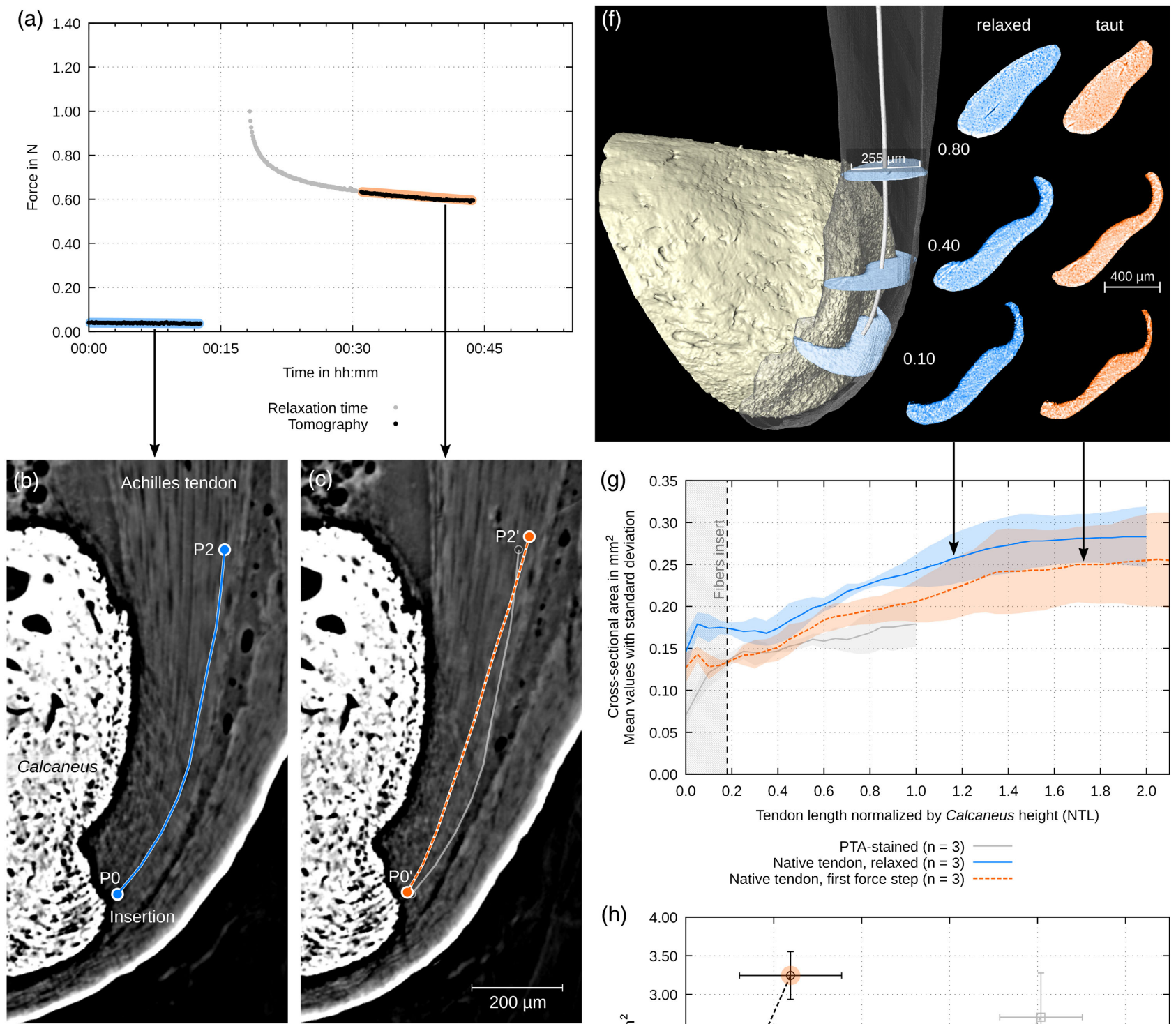

PTA-stained $(n=3)$
Native tendon, relaxed $(n=3)$ Native tendon, first force step $(n=3)$
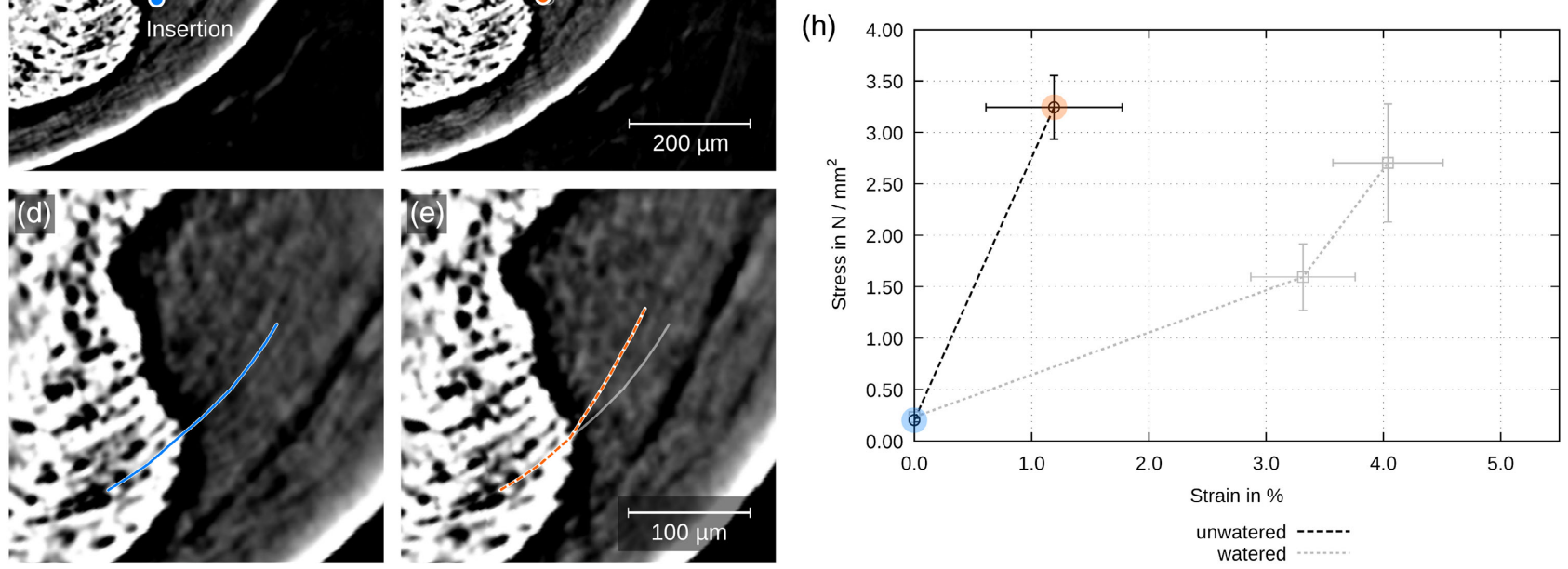

Figure 2. Comparison of relaxed (blue) and deformed specimens (red). A) Forces are logged during the relaxation time and the tomographies. B) Sections through a specimen in the relaxed state and at C) the first force level with the landmarks and the measuring distance used for strain measurement. D) Fiber course across the mineralization front in the relaxed state. E) The fibers are bent at the mineralization front at force level one. F) Cross sections are extracted for defined normalized positions along the tendon. G) The cross-sectional areas decrease under load. H) Diagram of stress over strain in unwatered and watered specimens (mean values with standard deviation, $n=3$ ).

of very small strains over the second force step. For the distal region the measured strains are negative at small absolute values with both methods. Overall, the strains measured by DVC tend to be smaller than the ones from the visual analysis. 

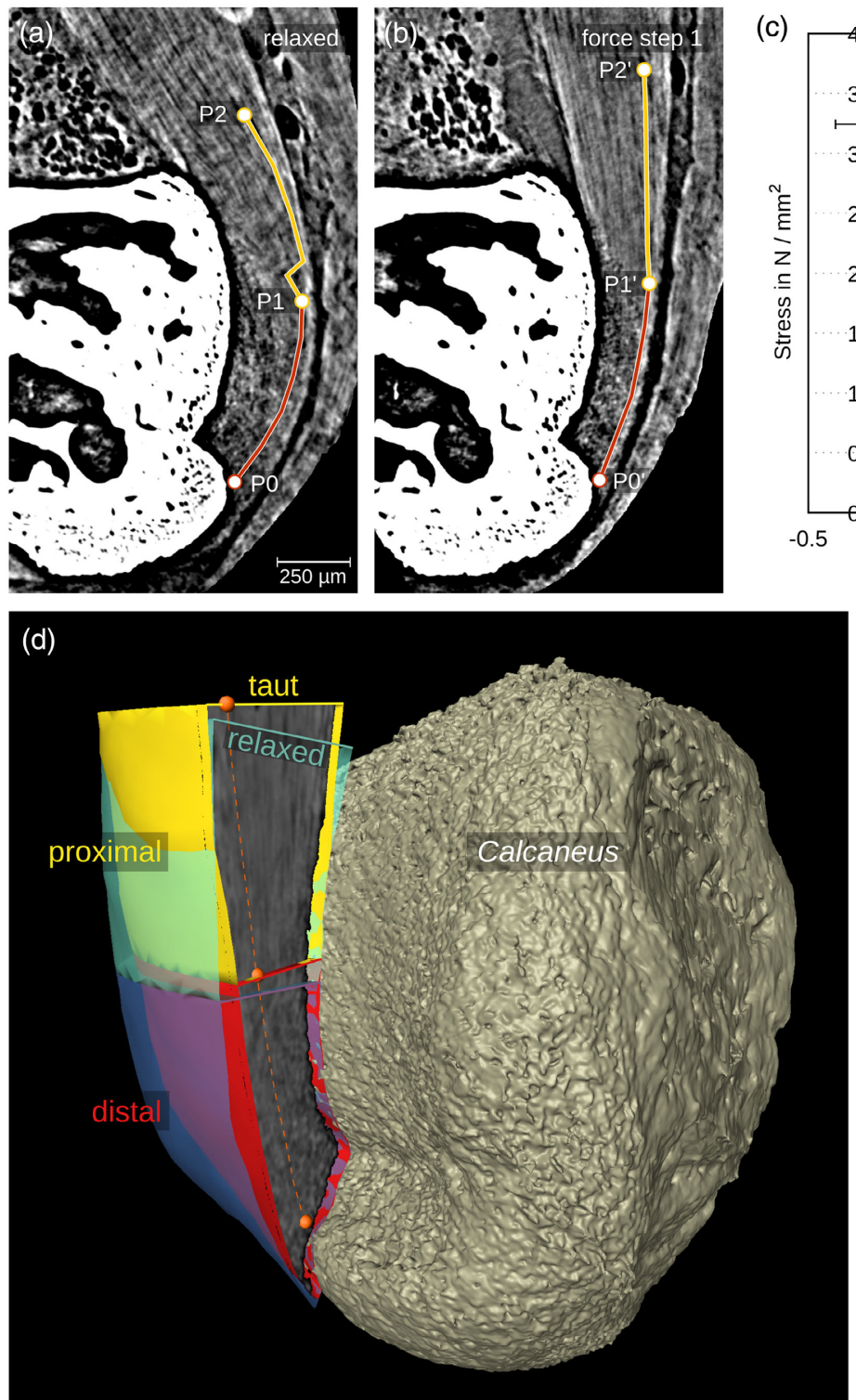

(c)

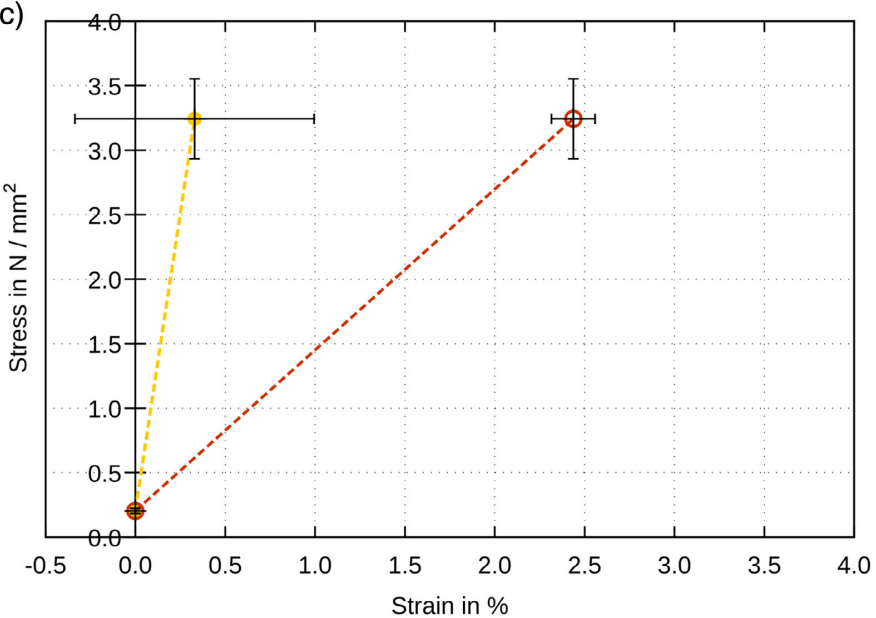

proximal --e--

distal --O--

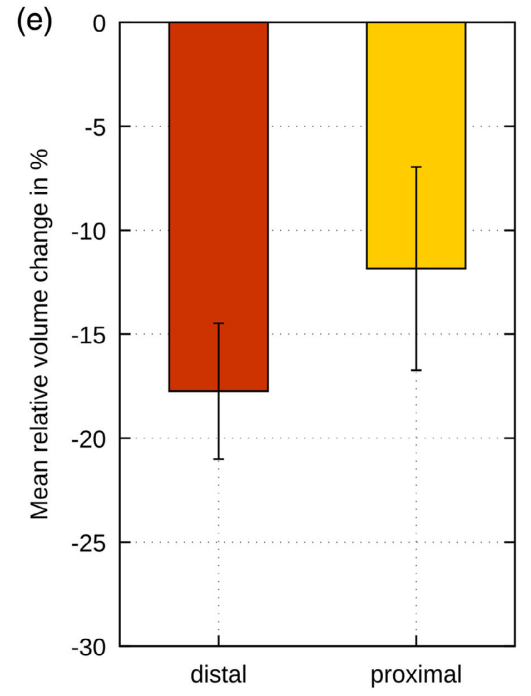

Figure 3. Differences between the distal tendon and the more proximal free tendon. A) Sections through a specimen in the relaxed state and B) at force level one with landmarks for the proximal (yellow) and distal (red) measuring distance. C) Diagram of stress over strain in the proximal and distal measuring distance (mean values with standard deviation, $n=3$ ). D) Rendering of the surfaces of the proximal and the distal segment of a specimen in the relaxed (cyan and purple) and stretched state (yellow and red). E) Diagram showing the volume changes in the proximal and the distal tendon segment with respect to the volume in the relaxed state (mean values with standard deviation, $n=3$ ).

\section{Discussion}

$\mathrm{SR} \mu \mathrm{CT}$ with propagation-based phase-contrast was proven useful as a technique for the visualization of the deformation inside intact entheses. Changes to the internal chemical environment of the specimens such as staining were not required. With a faster procedure for dissection and mounting examinations could also be carried out in fresh or thawn ex vivo specimens without a need to soak them in phosphate-buffered saline (PBS). PBS is known to lead to tendon swelling. ${ }^{[2]}$ Tomographic datasets were recorded within times short enough to preserve relevant material properties. The 3D structure of tendon tissue was resolved in sufficient detail for pattern tracking.

\subsection{The Effect of Water Content}

The CSA values measured in unwatered specimens demonstrate that the histological treatments used in our previous studies ${ }^{[40,41]}$ such as dehydration, demineralization, and critical-point drying, as well as PTA-staining, led to shrinkage and underestimation of the CSA. At the same time, watering seems to have led to swelling in this study. The tests in unwatered specimens are 

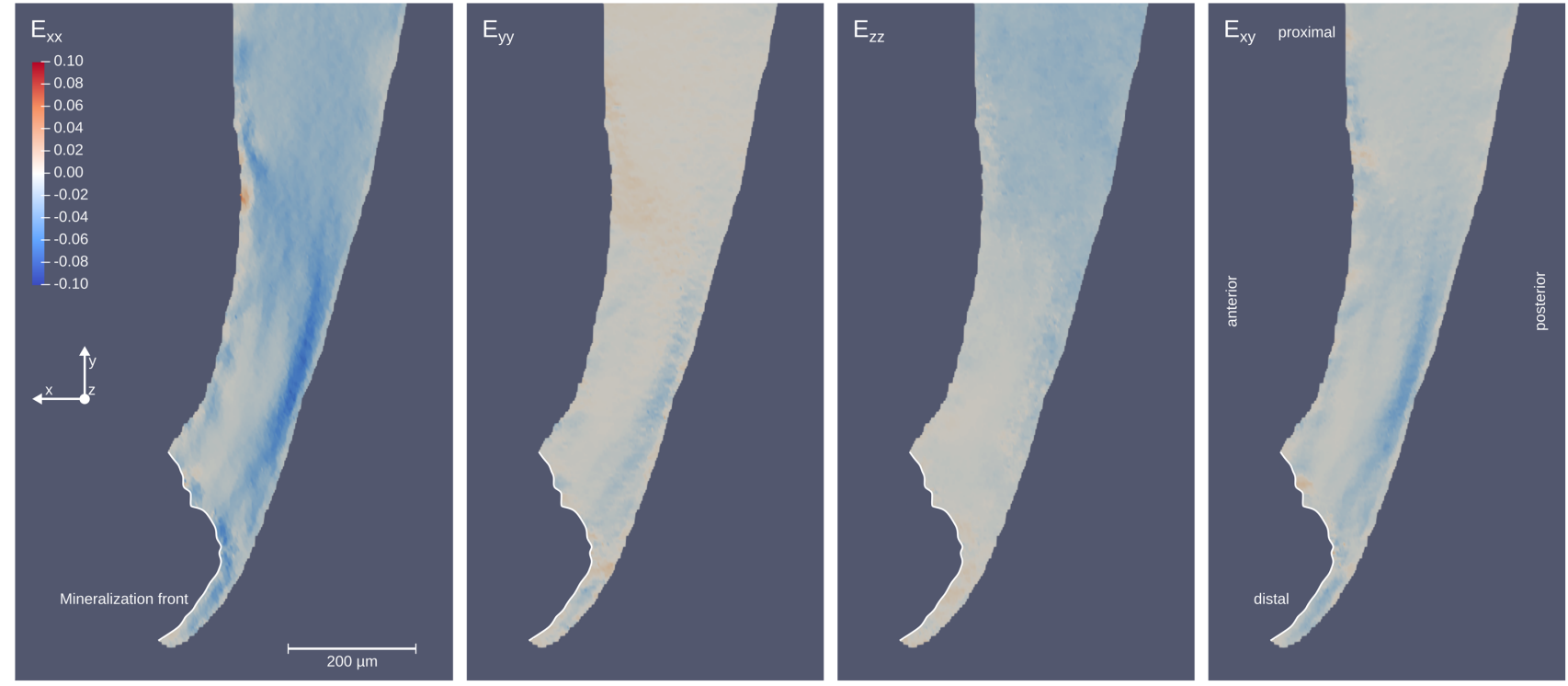

Figure 4. Strain fields for the second force step measured by digital volume correlation in an unwatered specimen. Several components of GreenLagrange strain tensor are plotted over the sagittal section of the tendon.

considered more reproducible. The water content of the specimens in future tests could be controlled by raising the relative humidity of the air within the specimen chamber to nearly $100 \%$ using a humidifier. ${ }^{[42]}$

\subsection{Tensile Behavior}

As seen from the increasing orientation of fibers, the first force step still comprises a part of the toe region. In contrast, the second force step is expected to reflect the linear region of the stress-strain relation.

Ansorge et al. ${ }^{[43]}$ examined the free Achilles tendon in mice aged 28 day in a PBS bath and published a toe modulus of $72.3 \pm 48.7 \mathrm{MPa}$, a strain of $1.91 \%$ at the transition from toe to linear region and a linear modulus of $544.0 \pm 240.8 \mathrm{MPa}$. Compared to this reference our study differs with regard to three conditions. 1) The age of the mice we examined is higher. Therefore, higher moduli and lower strains are expected to occur. 2) We used a much higher preloading, so that the stress-strain relation contains a smaller part of the toe region. 3) We did not determine the immediate relation of stress and strain, but the relation after a relaxation time. As reported, the stress after relaxation amounts to $60 \%$ of the stress measured at load application. Taking these considerations together, the stress-strain relation found in the unwatered specimens corresponds well to the values from the reference. Rinsing with water had a softening effect on the specimens. Therefore, the results from the watered specimens are less meaningful with regard to the in vivo behavior.

The higher age of the examined mice (C57BL/6 J) also explains the occurrence of tendon mineralizations. ${ }^{[4]}$ We do not expect the mineralizations to add substantial changes to the tensile behavior in the more distal parts of the tendon that were subject to the examinations. Connizzo and Grodzinsky ${ }^{[23]}$ report that differences between the supraspinatus tendon midsubstance and the insertion with regard to permeability and modulus are present in mature mice (aged 4 months) and disappear in aged mice (18 months).

\subsection{Regional Differences}

The existence of a distal region that is more compliant than either tendon or bone is supported by our findings of higher strains in the distal tendon of unwatered specimens.

Volume losses can be related to the Poisson's ratio of the tissue, but they can also result from compressive stress. The relative volume losses in the distal part of the specimens tend to be higher than in the proximal tendon. This is mainly attributed to the curvature of the tendon over the Tuber calcanei. Accordingly, tensile stress in the tendon leads to a compression of the distal tendon against the bone surface. We expect that the liquid that is pressed out accumulates in the bursae and plan to examine the volume changes in the bursae. The influence of compression could be examined by changing the insertion angle and accordingly the angle of load application. Such a study could as well address the question how the enthesis reacts to changes in the angle during locomotion.

The changes in volume also bring about changes in the angles of the fibers at the insertion to the hard tissues. The volume renderings confirm findings in sliced specimens by Sevick et al., ${ }^{[25]}$ who observed that fibers under load are bent in a narrow band of soft tissue adjacent to the mineralization front. This implicates very small curvature radii - and could lead to high local stress which is in contrast with previous ideas of enthesis function. ${ }^{[4,26,45]}$ In an unpublished series of radiographic projections, we observed that fibers are bent like that within seconds after load application, whereas the changes within the further relaxation time are small. However, the strain rate was much lower than in vivo rates even in these experiments. 
The initial hypothesis was that pressure in the liquid phase would have a higher influence on the mechanical behavior in intact specimens. Instead many findings from sliced specimens were confirmed, for example the presence of a compliant region and angular changes of the fibers at the insertion to hard tissue. The specimens were imaged after relaxation to avoid motion blur during the tomography. Volume losses and liquid accumulation in the bursae demonstrate that liquid movements take place during loading and relaxation. We still assume that examinations during load application and relaxation would find effects related to the pressurization of the liquid phase.

\section{Perspectives}

\subsection{Measuring Stress and Strain}

The tensile test of this study had some limitations. The cone used for muscle trapping allowed slippage which was compensated by local measurements of strain. If both ends of the specimen were clamped with a known compliance, an overall clamp-to-clamp strain could be calculated. An even better recording of the loading conditions could be achieved by introducing the complete test length into the field of view.

For the evaluation, we assumed a homogeneous distribution of stress and constant CSAs along the tendon. We nevertheless know that these gross estimations do not reflect the condition in the Achilles tendon in mice. ${ }^{[4]}$ Instead, stress could be estimated using finite element analyses in future studies.

Importantly, strain was measured along the fibers in the visual analysis. However, longitudinal sliding among fibers and shear of the matrix between them is a major mechanism of tendon deformation. ${ }^{[29]}$ Strain along the fibers relates to mechanisms such as uncrimping and sliding among fibrils. Following a simplifying assumption, these strains along fibers were used as an estimate of tissue strain and for the calculation of volume changes. It would be more meaningful to measure strain at a higher local resolution and relate strain tensors to the local orientation of fibers. Such an approach would enable the distinction of deformation mechanisms in 3D.

The differences between strain values from the visual analysis and the DVC analysis could be caused by inaccuracies of the visual approach. A more thorough evaluation including DVC over several force steps at larger sample sizes will be required to quantify the error. Nevertheless, both approaches lead to similar qualitative findings in the specimen examined indicating that the strains in the second force step are low. The distal part of the tendon is shortened at the position of the measuring distance in spite of an increase in force. The agreement of both methods supports that the measuring distance was factually shortened during force step two. Differences in the relaxation behavior along the tendon could have led to this phenomenon.

\subsection{X-Ray Radiation Dose}

The application of SR $\mu \mathrm{CT}$ in deformation studies of entheses is still restricted by the low number of tomographic datasets and thus force levels that can be recorded before the specimen fails.
The relaxation curves before specimen failure did not differ notably between the scanned specimens and the control. Instead, the specimens seem to have failed, when the X-ray radiation dose exceeded a critical level and when at the same time the mechanical load was either maintained or further increased. The observation of ruptures at the insertion hints to an unusual failure mechanism. The most common failure mechanisms of entheses are avulsion fractures and midsubstance ruptures. ${ }^{[46]}$ Measurements of the radiation dose are critical to relate the findings to the literature. ${ }^{[47]}$

Both, the X-ray radiation dose and the time under mechanical load, can be reduced by a minimization of tomography time. Recent developments at the beamline used show that durations of tomographies can be decreased to $15 \mathrm{~s}$. X-ray radiation dose can be further decreased by utilizing a fast X-ray shutter closing the beam path between single projections. The control experiment demonstrates that more than five force levels could be recorded under such conditions. Accordingly, the relation between strain and stress could be explored with smaller intervals between the force levels.

\subsection{Image Quality and Pattern Tracking}

The reliabilities of visual pattern tracking and DVC both depend on image quality. As yet, patterns that identify positions along the fibers are partly concealed by superimposed artifacts. Several parameters can contribute to an improvement of image quality. 1) The previously described reduction in the duration of tomographic data acquisition would at the same time decrease motion blur. 2) If motion blur is low, higher spatial resolutions could still increase the contrast between fibers and the nonfibrillar matrix. 3) The combination of photon energy, propagation distance and parameters for reconstruction and phase retrieval still requires further examination with regard to its effect on signal to noise ratio in the studied specimens. 4) Recent technological advancements in speckle-imaging and grating interferometry lead to an increase of resolution in grating based phase-contrast $\mathrm{SR} \mu \mathrm{CT}$ which is known to achieve a higher dynamic contrast range ${ }^{[36]}$ and a reduction of artifacts in specimens with heterogeneous absorbance. Grating-based phase-contrast additionally allows absolute quantitative evaluation. However, grating-based imaging leads to higher radiation doses. 5) Image quality can also be increased by post-processing of the reconstructed images and application of tools for artifact filtering.

The application of DVC to the second force step in one specimen yielded plausible results. A further evaluation of this approach seems to be worthwhile. The intervals between the force levels in this study led to large displacements of patterns between the relaxed state and the first force level and prevented the application of DVC to the complete deformation test. Shorter tomography times will facilitate the recording of more force levels before a critical radiation dose is reached.

Automatic tracking would improve the approach of this study in several ways. 1) It leads to a higher reproducibility of the measurements. 2) Displacements can be measured at a high spatial resolution and 3) over the complete region of interest. 4) 3D strain tensors can be calculated from the results. 


\subsection{Time-Dependent Phenomena}

Shorter tomography times also facilitate an onset of tomographies after a shorter relaxation time because they increase the tolerance for relaxation motions within the specimens. Gupta et al. hint to the fact that "a significant fraction of any load- or strain-induced changes occur within the first few seconds" [48] Even tomography times in the order of ten seconds appear long when it comes to the study of in vivo dynamics and tendon viscoelastic and poroelastic behavior. To reach subsecond temporal resolutions, tomographies should be combined with series of radiographic projections in a lateral or medial view to monitor parameters such as tendon thickness or the orientation of fibers near the insertion through loading and relaxation.

\section{Conclusion}

By a combination of a mechanical test and propagation-based phase-contrast SR $\mu \mathrm{CT}$ the 3D structure of Achilles tendons in mice was visualized at several defined force levels. Two findings of studies in slices were confirmed in intact specimens. 1) The region near the insertion was found to be more compliant than the free tendon. 2) The fibers near the transition to hard tissue are bent under load with respect to their orientation in hard tissue. Bending occurs within a relatively short length near the insertion.

Tomography times were identified as a central parameter to enable automatic pattern tracking. Recent developments at the beamline facilitate faster tomographies. Therefore, the combination of techniques tested is suitable for a wider application in the 3D soft tissue mechanics of small animals.

\section{Experimental Section}

Animals: The insertion of the Achilles tendon is a representative example of a fibrocartilaginous enthesis. Together with the sesamoid fibrocartilage, the periosteal cartilage, the bursa between them and Kager's fat pad protruding into the bursa, it embodies the idea of an enthesis organ. ${ }^{[49,50]}$ At the same time, it is accessible to dissection and imaging. In this study the Achilles tendon enthesis in mice (Figure 1A) is examined because it is small enough to fit into the field of view used for high resolutions at the $\mathrm{SR} \mu \mathrm{CT}$ beamline. Fiber size seems to show negative allometry with body size. ${ }^{[41,51]}$ Consequently, a better resolution of the fibers can be achieved in small mammals. From histological examinations, ${ }^{[40]}$ the distribution of collagen type II in rats ${ }^{[3]}$ and the allometry of tendon entheses, ${ }^{[52]}$ we expect that the unmineralized fibrocartilage and accordingly the compliant region occupies an $80 \mu \mathrm{m}$ wide band next to the mineralization front. Thus, resolving the compliant region seems feasible.

Hind limbs of mice from two strains (C57BL/6), NHE1-SMMHC 0) were obtained freshly within $15 \mathrm{~min}$ after euthanasia at Institut für Allgemeine Zoologie und Tierphysiologie of the Friedrich Schiller University Jena, as well as at the Service Unit for small rodents and the Animal Facility of the University Hospital Jena. The necessary approval for the handling of the animals was obtained by these institutes. The hind limbs were cryofixed either in isopentane cooled with liquid nitrogen or directly in liquid nitrogen. Six hindlimbs were evaluated and are reported in this article, one further hindlimb is used as a control for the influence of radiation. The hindlimbs stem from seven individuals from both sexes, aged 8-16 months that weighed between 30 and $37 \mathrm{~g}$. All procedures involving animals conformed to national and international ethical standards.
Tensile Testing Device: For imaging specimens under load a custom testing device was built (Figure 1B,C). In the path of the synchrotron X-ray beam a non-metal construction was required which was widely realized by $3 \mathrm{D}$ printing. An acrylic glass cylinder forms the walls of the specimen chamber. A clamp for the Calcaneus can be fixed to a holder at the base of the specimen chamber. A thread connects a hollow cone for trapping the M. triceps surae to a load cell above the specimen chamber (type 8523-5020 by Burster Präzisionsmesstechnik GmbH \& Co. KG, Gernsbach, Germany) with a nominal force of $20 \mathrm{~N}$. The thread runs over a pulley that is mounted on a ball bearing to minimize rotational friction. The angle between tendon and Calcaneus axis can be adjusted by relocating the axis of the ball bearing along a notch ("angle of load application"). Above the pulley, the thread forms a loop that is slipped over a hook at the load cell. The upper side of the load cell is connected to a precise linear actuator (type M-35 by Physik Instrumente $\mathrm{GmbH} \& \mathrm{Co}$. KG, Karlsruhe, Germany), that can displace it with a step size of below 1 micron. Load cell and actuator are integrated in a control loop ${ }^{[36]}$ so that a preset force can be approached automatically. The whole device is mounted on an $X Y$ table with two linear degrees of freedom (DOFs) for horizontal positioning. The $X Y$ table is itself mounted on the sample stage of a synchrotron beamline (P05, Petra III, DESY, Hamburg, Germany) operated by Helmholtz-Zentrum Geesthacht ${ }^{[53,54]}$ with one rotational DOF for scanning and one linear DOF in $z$-direction for vertical positioning.

Dissection and Mounting: The specimens were transferred to the imaging facility on dry ice. One specimen at a time was thawed in PBS freshly taken from a fridge, and subsequently dissected. The $M$. triceps surae was separated from the shank and the knee. The plantaris tendon was isolated from the Achilles tendon and cut at midlength. Keeping the Achilles tendon intact, the foot was separated from the shank at the ankle and placed in the clamp of the tensile testing device with its dorsal side pointing to the upper part and the posterior tip of the Calcaneus protruding from the tip of the clamp. The $M$. triceps surae was trapped in the hollow cone. A slit in the cone reaches to its tip. The Achilles tendon was introduced to the slit and gently pulled to the tip of the cone. Before mounting them into the tensile testing device, the clamp and the cone with the specimen were transferred to PBS again. We refrained from a continuous hydration of the specimen in a PBS bath because the higher attenuation would have decreased the contrast. The angle of load application was set to $90^{\circ}$. The factual angle of load application can deviate slightly from this value depending on the position of the Calcaneus along the clamp.

As the specimen chamber is nearly closed, the humidity in the chamber can be increased to reduce evaporation. Therefore, the floor of the specimen chamber was covered with foam and paper material soaked with deionized water. In the initial experiments the specimens were rinsed with deionized water to compensate for drying during the mounting process ("watered specimens"). However, this seemed to lead to swelling of the specimens. This step therefore was left out in later experiments ("unwatered specimens").

Mechanical Tests: In vivo measurements ${ }^{[55]}$ suggest that the forces transferred by the Achilles tendon can exceed $3 \mathrm{~N}$. In locomotion, forces occur dynamically and cyclically. The objective of the experiments was to examine the relation of stress and strain along the fibers in the free tendon and near the insertion. Because of the necessity to minimize motion within the specimen during the tomography, we had to examine each specimen in a quasi-static state after a relaxation phase. Measuring the load after relaxation is a common approach to the tensile behavior of tendons and their insertions..$^{[8,23,56]}$ The tissue cannot withstand forces as high as the in vivo forces in such a quasi-static experiment because the loads have to be applied for a longer period. ${ }^{[2]}$

Our main input parameters in the quasi-static experiments were the target force and the given relaxation time. However, stress does not only depend on the input force, but also on the tendon CSA and the relaxation behavior in each individual specimen. Accordingly, the applied stress varies between the specimens in spite of representing the "independent variable".

In each step of the force program, the stepping motor displacing the muscle cone was actuated until the target force value was measured by the load cell. The first tomographic dataset ("relaxed state") was recorded at a 
preloading of $0.05 \mathrm{~N}$. In the initial experiments, various values for the next force level were explored. In the unwatered specimens, that were thought to reflect the physiological state more accurately, the target force was consistently set to $1.0 \mathrm{~N}$. After reaching the target force, the displacement was maintained and the forces were logged over a relaxation time of 7-12 min. Afterwards, the second tomographic dataset was recorded ("force level one"). The stepping motor was actuated again to increase the force by a defined interval of either $0.2 \mathrm{~N}$ or $0.4 \mathrm{~N}$ above the first target force. After relaxation, a further tomographic dataset was recorded ("force level two"). This protocol was followed up to the failure of the specimen.

To control for the influence of radiation, the mechanical behavior is compared to a specimen that underwent the same force program but was not exposed to radiation during the tomography phase.

Imaging: The tensile testing device was mounted on the sample stage of the synchrotron beamline. Before the start of the mechanical testing program, the specimen was centered about the axis of rotation via the $X Y$ table. The height position of the sample stage was adjusted to place the insertion zone of the tendon at the lower end of the field of view.

The tomographies were carried out at a photon energy of $35 \mathrm{keV}$. Such a high photon energy is utilized to reduce the overall radiation dose a specimen is subjected to by a reduction in absorbance. Propagation-based phase contrast makes use of the generation of intensity contrast during the propagation of the transmitted wave front. The distance between specimen and detector is thus referred to as propagation distance. It was set to $0.80 \mathrm{~m}$. The optical magnification between the scintillator and the CMOSsensor was 9.96. The CMOS-sensor comprised a grid of 5120 times 3840 pixels with a linear pixel size of $6.40 \mu \mathrm{m} .{ }^{[57]}$ The resulting field of view spanned $3.29 \mathrm{~mm}$ times $2.47 \mathrm{~mm}$. During a tomography in standard mode 1201 to 1801 projections were recorded over an angular range of $180^{\circ}$. The number of projections was set to 1800 for the same angular range in fly-scan mode with a continuous rotation of the specimen. A tomography in standard mode took between 15 and $26 \mathrm{~min}$, while the tomography time in fly-scan mode was $13 \mathrm{~min}$. Each projection was taken with an exposure time of $350 \mathrm{~ms}$.

Data Analysis: Tomographic reconstruction has been done by applying a transport of intensity phase retrieval approach and using the filtered back projection algorithm (FBP) implemented in a custom reconstruction pipeline ${ }^{[58]}$ using Matlab (MATLAB ${ }^{\circledR}$ by MathWorks, Inc., Natick, Massachusetts, USA) and the Astra Toolbox ${ }^{[59-61]}$ The raw projections were binned two times for further processing. The resulting effective voxel size of the reconstructed tomographic volumes is $1.29 \mu \mathrm{m}$.

The volume images were loaded in a software for image manipulation and analysis (Image) 1.52p by National Institutes of Health, Bethesda, Maryland, USA). The histograms were cropped to the part of the range with high voxel counts, the bit depth was reduced to 16 bit. The volume images were cropped to the region of interest and loaded in a 3D image analysis software (Amira Software 6.3.0 by Thermo Fisher Scientific Inc., Waltham, Massachusetts, USA). The bone was marked by thresholding and region growing, and the bone surface was extracted. Pairs of landmarks were assigned to common patterns on the bone surfaces of the relaxed state as a reference and of each of the force levels. Based on these landmarks the volume image of each force level was aligned to the reference and subsequently resampled to the reference grid.

The main analysis of deformation was based on visual inspection. For comparison of two co-registered volume images from a specimen, a section plane parallel with the fibers was added. If the orientation of the fibers changed between the volume images, the plane was defined to intersect with three points: a position at the bone surface and a pair of positions that showed correspondence between the force levels. Such pairs of positions are connected to the position at the bone surface by fibers.

After aligning the section plane, it was used to search for corresponding patterns in three regions along the tendon and to mark them by pairs of landmarks: near the interface with the hard tissue (P0), at the transition from curved fibers in the distal tendon to straight fibers in the free tendon (P1) and in the more proximal free tendon (P2). Position P1 is defined according to the fiber curvature at force level one. The free tendon experienced large displacements between relaxed state and force level one. Therefore, suitable patterns for position P2 were searched for in a large area. Semilandmarks were placed between the landmarks according to the course of the tendon fibers. The semilandmarks do not reflect information about the lengthwise position along a fiber. We accounted for gross fiber kinks when positioning the semilandmarks (Figure 3A), but not for low wavelength crimps as the latter were not resolved in all cases. For each force step, the angle between the fiber orientations above and below the mineralization front was measured in the fiber course corresponding to position $\mathrm{P} 0$. The angular change between the force steps was calculated.

The coordinates of landmarks and semilandmarks were exported. A section of the tendon was oriented according to the coordinates of $\mathrm{P} 1$ and a normal vector that was calculated from the coordinates of the neighboring semilandmarks. The section was exported to determine tendon CSA. At each force level, the total length (P0-P2), the length of the distal (P0-P1) and the length of the proximal segment (P1-P2) were measured along the course determined by the semilandmarks using a custom software (Cloud2 version 14.11 .29 by Heiko Stark, https://starkrats.de). Linear strains were calculated from the measured lengths.

$\varepsilon=\left(I_{1}-I_{0}\right) / I_{0}$

Where $\varepsilon$ is the linear strain, $I_{0}$ is the length in the relaxed state, and $I_{1}$ is the length at the corresponding force level.

The mean force was calculated from the forces logged during tomography. Stress was calculated from the mean force and the tendon CSA at P1. Tangent moduli of the stress-strain curve were approximated by the slope of the line connecting neighboring data points.

The cross-sectional areas were measured in slices extracted from the tendon segment based on a previously established protocol. ${ }^{[40,41]}$ Accordingly, the positions of the slices are determined using a midtendon spline and normalization of lengths along this spline by Calcaneus height.

To measure the relative volume change during the force levels, the tendon was segmented in each volume image and exported as a surface. The coordinates of the landmarks $\mathrm{P} 1$ and $\mathrm{P} 2$ and the neighboring semilandmarks were used to define section planes. The tendon surfaces were cut into separate closed surfaces at these section planes. The volume enclosed by each of the resulting surfaces was measured using a 3D computer graphics software (Blender $2.79 \mathrm{~b}$ by Blender Foundation, Amsterdam, Netherlands). The relative volume changes were calculated from the volumes in the relaxed state and at each of the force levels.

DVC-based Strain Analysis: In one unwatered specimen, the change in tissue structure between force level one and two was sufficiently small to track tissue displacements automatically via DVC. The analysis was performed from dense motion estimates of the tendon, the Calcaneus and the surrounding soft tissue. The displacement fields were calculated under the assumption of grey value constancy using a custom global 3D variational optical flow solver implemented in CUDA for minimizing the DVC problem. Input data were filtered for noise and artifacts with an iterative non-local means filter ${ }^{[62]}$ and global image contrast was enhanced by histogram equalization. This preprocessing routine emphasizes the contrast of textures related to tendon fibers ensuring high quality tracking of deformations in the region of interest. Larger displacements were captured by applying the optical flow algorithm to an image pyramid and using an inner/outer iteration scheme. ${ }^{[63]}$ Best results in preserving flow discontinuities whereas interpolating untextured and artifact heavy regions were achieved by using anisotropic flow-driven regularization, ${ }^{[64]}$ an L1 penalty term, ${ }^{[63]}$ fourth-order centered finite differences for derivatives and cubic interpolation for warping. Resulting displacement vectors were mapped onto a tetrahedral mesh of the tendon for visualization purposes. The result was rotated for the calculation of the strain tensor to align the tendon orientation with the $y$-axis of the coordinate system and to place the $x$-axis in the sagittal plane. The $z$-axis correspondingly is normal to the sagittal plane. The tensor components of the Green-Lagrange strain tensor were calculated for the elements of the tetrahedral mesh with this coordinate system using ParaView (version 5.8 .0 by Sandia National Laboratories, Kitware Inc. and Los Alamos National Laboratory, all in the USA). The landmarks and semilandmarks that correspond to the relaxed state in the visual analysis were as well displaced using the 
displacement fields from the optical flow computation to extract strain values for comparison.

Statistics: Data from the three specimens in each of the groups ("watered," "unwatered") were summarized giving the mean value and the standard deviation. The samples were tested for normal distribution using the test by David et al. $(\alpha=0.05, n=3)$. The homogeneity of variances was examined using the $F$-test $\left(\alpha=0.05, \nu_{1}=\nu_{2}=3\right)$. The samples were tested for differences with regard to their central tendencies using Student's $t$-test $(\alpha=0.05, \nu=4)$, Welch's $t$-test $(\alpha=0.05)$, and the $U$-test $(\alpha=0.05)$ depending on the type of distribution and homogeneity of variances.

\section{Acknowledgements}

The authors thank Benjamin Naumann, George-Philipp Franz, Agustín Jorge Elias-Costa, and David Junghanns who worked with them during the imaging periods at the beamline, Alexander Hipp and Felix Beckmann for knowledgeable support during the assembly and operation of the experiments, Elisabeth Meier and Ms. Brinkmann for keeping the animals, Sabine Bischoff and Michael van der Wall for advice with regard to animal provision and animal rights, Dirk Arnold for recommendations with regard to tissue preparation, Martin S. Fischer, Jörg Bossert, Hartmut Witte, Cornelius Schilling and Christian Rode for the discussion of study design and mechanical interpretation. This study was supported by two grants from Deutsches Elektronen-Synchrotron (DESY: I-20160046 and I-20170614) to Martin S. Fischer and Julian Sartori. This research was supported in part through the Maxwell computational resources operated at DESY.

Open access funding enabled and organized by Projekt DEAL.

\section{Conflict of Interest}

The authors declare no conflict of interest.

\section{Data Availability Statement}

Research data are not shared in a repository. But the authors are willing to provide the data upon reasonable request to the corresponding authors.

\section{Keywords}

digital volume correlation, hard-soft transition, multiscale tendon mechanics, propagation-based phase contrast, soft tissue imaging, synchrotron radiation-based microcomputed tomography

Received: January 23, 2021 Revised: June 20, 2021 Published online: July 26, 2021

[1] S. J. Mellon, K. E. Tanner, Int. Mater. Rev. 2012, 57, 235.

[2] R. Ker, Int. J. Fatigue 2007, 29, 1001.

[3] M. Benjamin, J. R. Ralphs, J. Anat. 1998, 193, 481.

[4] M. Benjamin, J. R. Ralphs, Ital. J. Anat. Embryol. 2000, 106, 151.

[5] S. Thomopoulos, G. R. Williams, J. A. Gimbel, M. Favata, L. J. Soslowsky, J. Orthop. Res. 2003, 21, 413.

[6] A. G. Schwartz, J. D. Pasteris, G. M. Genin, T. L. Daulton, S. Thomopoulos, PLoS ONE 2012, 7, 48630

[7] A. C. Deymier, Y. An, J. J. Boyle, A. G. Schwartz, V. Birman, G. M. Genin, S. Thomopoulos, A. H. Barber, Acta Biomater. 2017, 65, 25.
[8] L. Rossetti, L. A. Kuntz, E. Kunold, J. Schock, K. W. Müller, H. Grabmayr, J. Stolberg-Stolberg, F. Pfeiffer, S. A. Sieber, R. Burgkart, A. R. Bausch, Nat. Mater. 2017, 16, 664.

[9] L. Zhao, A. Thambyah, N. D. Broom, J. Anat. 2014, 224, 624.

[10] M. J. Bey, H. K. Song, F. W. Wehrli, L. J. Soslowsky, J. Orthop. Res. 2002, 20, 869

[11] S. Rigozzi, R. Muller, J. G. Snedeker, J. Biomech. 2009, 42, 1547.

[12] K. S. Miller, B. K. Connizzo, L. J. Soslowsky, Ann. Biomed. Eng. 2012, 40, 1102.

[13] K. S. Miller, L. Edelstein, B. K. Connizzo, L. J. Soslowsky, J. Biomech. Eng. 2012, 134, 031007.

[14] K. F. Mallett, E. M. Arruda, Acta Biomater. 2017, 56, 44.

[15] C. M. Luetkemeyer, L. Cai, C. P. Neu, E. M. Arruda, Extreme Mech. Lett. 2018, 19, 7 .

[16] D. C. Stouffer, D. L. Butler, D. Hosny, J. Biomech. Eng. 1985, 107, 158.

[17] D. F. Villegas, J. A. Maes, S. D. Magee, T. L. H. Donahue, J. Biomech. 2007, 40, 2655.

[18] S. P. Lake, K. S. Miller, D. M. Elliott, L. J. Soslowsky, J. Orthop. Res. 2009, 27, 1596.

[19] H. Sano, Y. Saijo, S. Kokubun, J. Shoulder Elbow Surg. 2006, 15, 743.

[20] R. C. Locke, J. M. Peloquin, E. A. Lemmon, A. Szostek, D. M. Elliott, M. L. Killian, J. Biomech. Eng. 2017, 139, 111007.

[21] H. Ahmadzadeh, B. R. Freedman, B. K. Connizzo, L. J. Soslowsky, V. B. Shenoy, Acta Biomater. 2015, 22, 83.

[22] B. K. Connizzo, A. J. Grodzinsky, J. Biomech. 2017, 54, 11.

[23] B. K. Connizzo, A. J. Grodzinsky, J. Biomech. Eng. 2018, 140, 051002.

[24] T. A. Wren, G. S. Beaupre, D. R. Carter, J. Rehabil. Res. Dev. 2000, 37, 135

[25] J. L. Sevick, Z. Abusara, S. H. Andrews, M. Xu, S. Khurshid, J. Chatha, D. A. Hart, N. G. Shrive, J. Orthop. Res. 2018, 36, 2506.

[26] H. Schneider, Anat. Embryol. (Berl.) 1956, 119, 431.

[27] N. J. Giori, G. S. Beaupré, D. R. Carter, J. Orthop. Res. 1993, 11, 581.

[28] J. P. Spalazzi, J. Gallina, S. D. Fung-Kee-Fung, E. E. Konofagou, H. H. Lu, J. Orthop. Res. 2006, 24, 2001.

[29] H. R. C. Screen, D. L. Bader, D. A. Lee, J. C. Shelton, Strain 2004, 40, 157.

[30] W. M. Han, S.-J. Heo, T. P. Driscoll, J. F. Delucca, C. M. McLeod, L. J. Smith, R. L. Duncan, R. L. Mauck, D. M. Elliott, Nat. Mater. 2016, 15, 477.

[31] E. E. Hoover, J. A. Squier, Nat. Photonics 2013, 7, 93.

[32] J. Buytaert, J. Goyens, D. De Greef, P. Aerts, J. Dirckx, Microsc. Microanal. 2014, 20, 1208.

[33] G. Kerckhofs, S. Stegen, N. Van Gastel, A. Sap, G. Falgayrac, G. Penel, M. Durand, F. Luyten, L. Geris, K. Vandamme, in Proc. IEEE Int. Symp. on Biomedical Imaging, IEEE, Piscataway, NJ 2016.

[34] G. R. S. Naveh, V. Brumfeld, M. Dean, R. Shahar, S. Weiner, Connect. Tissue Res. 2014, 55, 52.

[35] G. Tozzi, M. Peña Fernández, S. Davis, A. Karali, A. P. Kao, G. Blunn, Materials 2020, 13, 2579.

[36] J. Moosmann, D. C. F. Wieland, B. Zeller-Plumhoff, S. Galli, D. Krüger, A. Ershov, S. Lautner, J. Sartori, M. Dean, S. Köhring, H. Burmester, T. Dose, N. Peruzzi, A. Wennerberg, R. WillumeitRömer, F. Wilde, P. Heuser, J. U. Hammel, F. Beckmann, in Proc. SPIE 11113(Eds: B. Müller, G. Wang), International Society for Optics and Photonics, San Diego, CA 2019, p. 41.

[37] C. M. Disney, A. Eckersley, J. C. McConnell, H. Geng, A. J. Bodey, J. A. Hoyland, P. D. Lee, M. J. Sherratt, B. K. Bay, Acta Biomater. 2019, 92, 290

[38] C. M. Disney, P. D. Lee, J. A. Hoyland, M. J. Sherratt, B. K. Bay, J. Microsc. 2018, 272, 165

[39] K. Madi, K. A. Staines, B. K. Bay, B. Javaheri, H. Geng, A. J. Bodey, S. Cartmell, A. A. Pitsillides, P. D. Lee, Nat. Biomed. Eng. 2020, 4, 343. 
[40] J. Sartori, S. Köhring, H. Witte, M. S. Fischer, M. Löffler, J. Anat. 2018, 233, 370.

[41] J. Sartori, H. Stark, Acta Biomater. 2021, 120, 146.

[42] L. Bailly, T. Cochereau, L. Orgéas, N. Henrich Bernardoni, S. Rolland du Roscoat, A. McLeer-Florin, Y. Robert, X. Laval, T. Laurencin, P. Chaffanjon, B. Fayard, E. Boller, Sci. Rep. 2018, 8, 14003.

[43] H. L. Ansorge, S. Adams, D. E. Birk, L. J. Soslowsky, Ann. Biomed. Eng. 2011, 39, 1904

[44] E. J. O. O'Brien, C. B. Frank, N. G. Shrive, B. Hallgrímsson, D. A. Hart, Int. J. Exp. Pathol. 2012, 93, 319.

[45] M. Benjamin, E. J. Evans, L. Copp, J. Anat. 1986, 149, 89.

[46] J. Gao, T. Rasanen, J. Persliden, K. Messner, J. Anat. 1996, 189, 127.

[47] H. D. Barth, E. A. Zimmermann, E. Schaible, S. Y. Tang, T. Alliston, R. O. Ritchie, Biomaterials 2011, 32, 8892.

[48] H. S. Gupta, J. Seto, S. Krauss, P. Boesecke, H. R. C. Screen, J. Struct. Biol. 2010, 169, 183.

[49] M. Benjamin, D. McGonagle, J. Anat. 2001, 199, 503.

[50] J. Canoso, J. Rheumatol. 1998, 25, 1254.

[51] G. G. Handsfield, L. C. Slane, H. R. C. Screen, J. Biomech. 2016, 49, 3122.

[52] A. C. Deymier-Black, J. D. Pasteris, G. M. Genin, S. Thomopoulos, J. Biomech. Eng. 2015, 137, 111005(8).

[53] I. Greving, F. Wilde, M. Ogurreck, J. Herzen, J. U. Hammel, A. Hipp, F. Friedrich, L. Lottermoser, T. Dose, H. Burmester, M. Müller, F. Beckmann, in Proc. SPIE 9212, Developments in X-Ray Tomography IX (Ed: S. R. Stock), San Diego, CA 2014, p. 921200, https://doi.org/10.1117/12.2061768.

[54] F. Wilde, M. Ogurreck, I. Greving, J. U. Hammel, F. Beckmann, A. Hipp, L. Lottermoser, I. Khokhriakov, P. Lytaev, T. Dose,
H. Burmester, M. Müller, A. Schreyer, AIP Conference Proceedings, New York, NY 2016, 1741, p. 030035, https://doi.org/10.1063/1. 4952858.

[55] J. A. Ashton-Miller, Y. He, V. A. Kadhiresan, D. A. McCubbrey, J. A. Faulkner, J. Appl. Physiol. 1992, 72, 1205.

[56] S. E. Szczesny, D. M. Elliott, Acta Biomater. 2014, 10, 2582.

[57] P. Lytaev, A. Hipp, L. Lottermoser, J. Herzen, I. Greving, I. Khokhriakov, S. Meyer-Loges, J. Plewka, J. Burmester, M. Caselle, M. Vogelgesang, S. Chilingaryan, A. Kopmann, M. Balzer, A. Schreyer, F. Beckmann, in Proc. SPIE 9212, Developments in X-Ray Tomography IX (Ed: S. R. Stock), San Diego, CA 2014, p. 921218, https://doi.org/10.1117/12.2061389.

[58] J. Moosmann, A. Ershov, V. Weinhardt, T. Baumbach, M. S. Prasad, C. LaBonne, X. Xiao, J. Kashef, R. Hofmann, Nat. Protoc. 2014, 9, 294.

[59] W. J. Palenstijn, K. J. Batenburg, J. Sijbers, J. Struct. Biol. 2011, 176, 250.

[60] W. van Aarle, W. J. Palenstijn, J. De Beenhouwer, T. Altantzis, S. Bals, K. J. Batenburg, J. Sijbers, Ultramicroscopy 2015, 157, 35.

[61] W. van Aarle, W. J. Palenstijn, J. Cant, E. Janssens, F. Bleichrodt, A. Dabravolski, J. De Beenhouwer, K. Joost Batenburg, J. Sijbers, Opt. Express 2016, 24, 25129.

[62] S. Bruns, S. L. S. Stipp, H. O. Sørensen, Adv. Water Resour. 2017, 105, 96.

[63] T. Brox, A. Bruhn, N. Papenberg, J. Weickert, in Computer. Vision ECCV 2004 (Eds.: T. Pajdla, J. Matas), Springer, Berlin, Heidelberg 2004, p. 25.

[64] J. Weickert, C. Schnörr, Int. J. Comput. Vis. 2001, 45, 245. 